\title{
Well Ordered Monotone Iterative Technique for Nonlinear Second Order Four Point Dirichlet BVPs
}

\author{
Amit K. Verma and Nazia Urus \\ Department of Mathematics, Indian Institute of Technology Patna \\ Bihta, 801103 Patna, India \\ E-mail(corresp.): akverma@iitp.ac.in \\ E-mail: naziaurus2011@gmail.com
}

Received January 11, 2021; revised November 15, 2021; accepted November 15, 2021

\begin{abstract}
In this article, we develop a monotone iterative technique (MI-technique) with lower and upper (L-U) solutions for a class of four-point Dirichlet nonlinear boundary value problems (NLBVPs), defined as,

$$
-\psi^{\prime \prime}(x)=F\left(x, \psi, \psi^{\prime}\right), \quad 0<x<1, \mathrm{BCs}(i) \equiv \psi(i)-c_{i} \psi\left(\eta_{i}\right)=0, \quad i=0,1,
$$

where $0<c_{0}<1, c_{1}>0,0<\eta_{0} \leq \eta_{1}<1, \psi(x) \in C^{2}[0,1]$, the non linear term $F\left(x, \psi, \psi^{\prime}\right)$ is continuous function in $x$, one sided Lipschitz in $\psi$ and Lipschitz in $\psi^{\prime}$. To show the existence result, we construct Green's function and iterative sequences for the corresponding linear problem. We use quasilinearization to construct these iterative schemes. We prove maximum principle and establish monotonicity of sequences of lower solution $\left(l_{m}(x)\right)_{m}$ and upper solution $\left(u_{m}(x)\right)_{m}$ such that $l_{m}(x) \leq u_{m}(x)$, $\forall m \in \mathbb{N}$. Then under certain sufficient conditions we prove that these sequences converge uniformly to the solution $\psi(x)$ in a specific region where $\frac{\partial F}{\partial \psi} \neq 0$.
\end{abstract}

Keywords: Green's function, monotone iterative technique, maximum principle.

AMS Subject Classification: 34B05; 34B10; 34B15.

\section{Introduction}

In real-life, there are many applications of multi-point ( $m$-point) boundary value problems (BVPs), e.g., suspension bridge. Two-point BVPs conduct small size bridges, in order to conduct large size bridges we need $m$-point boundary conditions (BCs) [15]. Lazer and McKenna [21] have discussed the

Copyright (C) 2022 The Author(s). Published by Vilnius Gediminas Technical University

This is an Open Access article distributed under the terms of the Creative Commons Attribution License (http://creativecommons.org/licenses/by/4.0/), which permits unrestricted use, distribution, and reproduction in any medium, provided the original author and source are credited. 
existence and multiplicity of periodic solutions of possible mathematical models for the nonlinear behavior of suspension bridge.

The study of $m$-point BVPs have become a broad area of research due to their wide applications. The $m$-point linear second-order BVPs was first studied by II'in and Moiseev [18]. In this article, we have focused on the existence of solution of second-order four-point Dirichlet NLBVPs. To study the existence, various methods are introduced such as fixed point (FP) index theory, MI-technique, shooting method, etc., we refer the reader to $[7,8,19,20]$. There are various techniques to study the following class of four-point Dirichlet NLBVPs,

$$
\begin{array}{r}
\psi^{\prime \prime}(x)+\beta q(x) F\left(x, \psi, \psi^{\prime}\right)=0, \quad 0<x<1, \\
\operatorname{BCs}(i) \equiv \psi(i)-c_{i} \psi\left(\eta_{i}\right)=0, \quad i=0,1 .
\end{array}
$$

Verma et al. [28] established MI-technique with L-U solution to study the existence of solution, where $\beta=q(x)=1$ and $c_{0}=0$. Also article [30], deals with the existence and multiplicity of positive solutions of above NLBVPs (1.1)(1.2), here $\psi^{\prime}$ is involved in $F$ explicitly and $\beta=q(x)=1$. For this, they have used Krasnoselskii FP theorem and triple FP theorem. By using FP theorem Liu et al. [22], studied the existence of positive solutions for second order problem $\psi^{\prime \prime}(x)+a(x) f(\psi(x))=0$ with BCs (1.2).

In article [4], authors concentrated on the existence, nonexistence, and multiplicity of positive solutions for the non resonance problem (1.1)-(1.2), where $F$ is independent of $\psi^{\prime}$. The main techniques used are the FP index theory, the Leray-Schauder degree, and the L-U solutions method. They have determined the range of $\beta$, for which there exists at least one, at least two, and no positive solution. The $m$-point Dirichlet NLBVPs, with different techniques, can also be seen in $[16,17,24]$. Bai et al. $[4,5,6]$ considered the NLBVPs (1.1)-(1.2), where $0<\eta_{0}, \eta_{1}<1,0 \leq c_{0}<\frac{1}{1-\eta_{0}}, 0<c_{1}<\frac{1}{\eta_{1}}$, and $q:(0,1) \rightarrow[0, \infty), F:[0,1] \times[0, \infty) \times \mathbb{R} \rightarrow[0, \infty)$ are non-negative continuous functions. Article [5], dealt with the non resonance problem where $\beta=1$ with $\Gamma \equiv c_{0} \eta_{0}\left(1-c_{1}\right)+\left(1-c_{0}\right)\left(1-c_{1} \eta_{1}\right)>0$. By using a new FP theorem, the authors established some multiplicity results. The article [6] dealt with the resonance problem where the nonlinear term does not depend on the first-order derivative. Here $\beta=1=q(x)=1$ with $\Gamma=0$. The authors established the $\mathrm{L}-\mathrm{U}$ solution method to obtain some existence and multiplicity results.

MI-technique was first introduced by E. Picard in 1890 [25]. By using MItechnique we can obtain existence results for a large class of BVPs. We study this technique to ensure the existence and approximation of solutions lying in a pair of ordered functions called L-U solutions. This technique also gives a constructive way to find the maximal and minimal solutions corresponding to the L-U solutions $[9,10,11,12]$. To know more about history and development related to MI-technique one can refer [14,27].

In this article, to study the existence of a solution we have explored an iterative process for a class of four-point Dirichlet NLBVPs with nonlinear 
source term, defined as follows,

$$
\begin{aligned}
-\psi^{\prime \prime}(x) & =F\left(x, \psi, \psi^{\prime}\right), \quad 0<x<1, \\
\operatorname{BCs}(i) & \equiv \psi(i)-c_{i} \psi\left(\eta_{i}\right)=0, \quad i=0,1,
\end{aligned}
$$

where $0<c_{0}<1, c_{1}>0,0<\eta_{0} \leq \eta_{1}<1, \psi(x) \in C^{2}[0,1]$, the non linear term $F\left(x, \psi, \psi^{\prime}\right)$ is continuous function in $x$, one sided Lipschitz in $\psi$ and Lipschitz in $\psi^{\prime}$. The iterative schemes we have constructed in this article are defined in (4.2)-(4.3), where $\lambda$ is non zero real number.

In [23], by using the FP of strict-set-contractions, Liu et. al. studied the existence of at least one or two positive solutions to the four-point BVPs (1.1). Various NLBVPs have been studied with multi-point BCs by using MI-technique. In 2019 [29], we have discussed the existence of solution for four-point NLBVPs (1.1) with BCs $\psi^{\prime}(0)=0$ and $\psi(1)=c_{0} \psi\left(\eta_{0}\right)+c_{1} \psi\left(\eta_{1}\right)$. We also have discussed the existence of solution of the above NLBVPs (1.1) in [26], for the same BCs where $F$ is independent of $\psi^{\prime}$. For the existence of solutions on three-point NLBVPs with MI-technique, one can refer [28]. For higher order BVP with four point BCs one can refer the work in $[1,2,3]$.

The work of this paper generalises our earlier work [28] and complement a recent paper [29]. Cherpion in his article [13], stated that the iterative scheme (4.2)-(4.3) do not work for constant $\lambda$. Also they stated that due to lack of uniform antimaximum principle it is impossible to develop MI-technique for the case $l_{m}(x) \geq u_{m}(x)$. This statement is true for our case also. The work in this paper is novel in the sense that even with constant $\lambda$ we are able to generate monotone sequences. Also, our results are based on simple assumptions, hence it can deal with larger class of nonlinear four point BVPs, e.g., we don't require sign restrictions [27, p. 27]. All these further approves the fact that L-U solution technique related to MI-method is most powerful technique to solve class of nonlinear BVPs [31].

This paper is divided into five sections. In the second section, we discuss some preliminaries. In the third section, we describe Green's function, solution of the corresponding linear problem, and maximum principle which is used to construct monotone L-U solutions. In the fourth section, L-U solutions are defined, some assumptions are considered on the source term $F$, and we establish the main result on the existence of a solution for $\lambda \neq 0$. The final section verifies the theoretical results numerically.

\section{Preliminaries}

In this section, we discuss linearization corresponding to the NLBVPs (1.1), and derive some important inequalities for $\lambda \neq 0$. The corresponding linear non homogeneous Dirichlet BVPs is as follows,

$$
-\psi^{\prime \prime}(x)-\lambda \psi(x)=q(x), \quad \operatorname{BCs}(0)=0, \quad \operatorname{BCs}(1)=A, \quad 0<x<1,
$$

where $\operatorname{BCs}(i), i=0,1$, is defined in (1.1), $q(x)=F\left(x, \psi, \psi^{\prime}\right)-\lambda \psi(x), \lambda \neq 0$, and $A$ has any constant value. 
Lemma 1. If $\lambda \in I_{0}=\left(0, \pi^{2} / 4\right), \delta_{1} \in \mathbb{R}^{+}$such that $\left(\lambda-\delta_{1}\right) \leq 0$, and there is a function $\delta_{2}:[0,1] \rightarrow[0, \infty)$ such that $\delta_{2}(0)=0$ and $\delta_{2}^{\prime}(x) \geq 0$, then

$$
\begin{aligned}
\text { (a). } & \left(\lambda-\delta_{1}\right) \sin \sqrt{\lambda} x+\delta_{2}(x) \sqrt{\lambda} \cos \sqrt{\lambda} x \leq 0, \text { if } \\
& \left(\lambda-\delta_{1}\right)+\sup _{x \in[0,1]} \delta_{2}^{\prime}(x) \leq 0, \forall x \in[0,1], \\
(b) . & \left(\lambda-\delta_{1}\right) \cos \sqrt{\lambda} x+\delta_{2}(x) \sqrt{\lambda} \sin \sqrt{\lambda} x \leq 0, \text { if } \\
& \left(\lambda-\delta_{1}\right) \cos \sqrt{\lambda}+\delta_{2}(1) \sqrt{\lambda} \sin \sqrt{\lambda} \leq 0, \quad \forall x \in[0,1] .
\end{aligned}
$$

Proof. For the proof of this lemma we refer [28].

Lemma 2. If $\lambda \in(-\infty, 0), \delta_{1} \in \mathbb{R}^{+}$such that $\left(\lambda+\delta_{1}\right) \leq 0$, and there is a function $\delta_{2}:[0,1] \rightarrow[0, \infty)$ such that $\delta_{2}(0)=0$, then

$$
\begin{aligned}
(a) . & \left(\lambda+\delta_{1}\right) \cosh (x \sqrt{|\lambda|})+\delta_{2}(x) \sqrt{|\lambda|} \sinh (x \sqrt{|\lambda|}) \leq 0, \quad \text { if } \\
& \left(\lambda+\delta_{1}\right)+\sup _{x \in[0,1]} \delta_{2}(x) \sqrt{|\lambda|} \leq 0, \quad \forall x \in[0,1] . \\
(b) . & \left(\lambda+\delta_{1}\right) \sinh (x \sqrt{|\lambda|})+\delta_{2}(x) \sqrt{|\lambda|} \cosh (x \sqrt{|\lambda|}) \leq 0, \quad \text { if } \\
& \left(\lambda+\delta_{1}\right)+\sup _{x \in[0,1]}\left(\delta_{2}^{\prime}(x)+\delta_{2}(x) \sqrt{|\lambda|}\right) \leq 0, \quad \forall x \in[0,1] .
\end{aligned}
$$

Proof. For the proof of this lemma we refer [28].

\section{Green's function and maximum principle}

In this section, we state two lemmas where we obtain Green's function for the BVPs (2.1), and then we show that under some assumptions the Green's function is non-positive. We also obtain the solution for the BVPs (2.1). Further we prove some important inequalities and establish the maximum principle which are used to construct monotone sequences of L-U solutions.

Assume that the following conditions hold;

$$
\begin{aligned}
\left(P_{0}\right): \lambda \in I_{0}, D_{\lambda^{+}}>0, A_{1}(\lambda)<0, A_{2}(\lambda) \leq 0, \text { where } \\
D_{\lambda^{+}}=\sqrt{\lambda}\left(c_{1} \sin \left(\left(\eta_{0}-1\right) \sqrt{\lambda}\right)-c_{1}\left(c_{1} \sin \left(\left(\eta_{0}-\eta_{1}\right) \sqrt{\lambda}\right)+\sin \left(\eta_{1} \sqrt{\lambda}\right)\right)\right. \\
\quad+\sqrt{\lambda} \sin (\sqrt{\lambda})), \quad A_{1}(\lambda)=c_{1} \sin \left(\eta_{1} \sqrt{\lambda}\right)-\sin (\sqrt{\lambda}), \text { and } \\
\quad A_{2}(\lambda)=\cos (\sqrt{\lambda})-c_{1} \cos \left(\eta_{1} \sqrt{\lambda}\right) . \\
\left(N_{0}\right): \quad \lambda \in(-\infty, 0), B_{1}(\lambda) \geq 0, B_{2}(\lambda)<0, B_{3}(\lambda)>0, B_{1}(\lambda)+B_{2}(\lambda)<0 \\
D_{\lambda^{-}}=\sqrt{|\lambda|}\left(c_{0} \sinh \left(\left(\eta_{0}-1\right) \sqrt{|\lambda|}\right)-c_{1}\left(c_{0} \sinh \left(\left(\eta_{0}-\eta_{1}\right) \sqrt{|\lambda|}\right)\right.\right. \\
\left.\left.\quad+\sinh \left(\eta_{1} \sqrt{|\lambda|}\right)\right)+\sinh (\sqrt{|\lambda|})\right), \quad B_{1}(\lambda)=\cosh (\sqrt{|\lambda|}) \\
\quad-c_{1} \cosh \left(\eta_{1} \sqrt{|\lambda|}\right), \quad B_{2}(\lambda)=c_{1} \sinh \left(\eta_{1} \sqrt{|\lambda|}\right)-\sinh (\sqrt{|\lambda|}) \\
B_{3}(\lambda)=1-c_{0} \cosh \left(\eta_{0} \sqrt{|\lambda|}\right) .
\end{aligned}
$$


Under the assumed sign restriction on $B_{1}, B_{2}$ and $B_{3}$, it is easy to deduce that $D_{\lambda^{-}}>0$.

Lemma 3. The Green's function of the following linear BVPs

$$
\psi^{\prime \prime}(x)+\lambda \psi(x)=0, \quad B C s(i)=0, \quad i=0,1, \quad 0<x<1,
$$

where $B C s(i)$ is defined in (1.1), is given as,

Case-1: If $\lambda \in I_{0}=\left(0, \pi^{2} / 4\right)$ then,

$$
\begin{aligned}
& \xi^{+}(x, t)=\frac{1}{D_{\lambda^{+}}} \\
& \times\left\{\begin{array}{l}
\sin (\sqrt{\lambda} x)\left(\sin (\sqrt{\lambda}(t-1))-c_{1} \sin \left(\sqrt{\lambda}\left(t-\eta_{1}\right)\right)\right) \\
+c_{0}\left(\sin \left(\left(\eta_{0}-1\right) \sqrt{\lambda}\right)-c_{1} \sin \left(\left(\eta_{0}-\eta_{1}\right) \sqrt{\lambda}\right)\right) \sin (\sqrt{\lambda}(t-x)) \\
\quad 0 \leq x \leq t \leq \eta_{0} ; \\
\sin (\sqrt{\lambda} t)\left(\sin (\sqrt{\lambda}(x-1))-c_{1} \sin \left(\sqrt{\lambda}\left(x-\eta_{1}\right)\right)\right), 0 \leq t \leq x \leq \eta_{0} ; \\
\quad \sin (\sqrt{\lambda}(t-1))-c_{1} \sin \left(\sqrt{\lambda}\left(t-\eta_{1}\right)\right)(\sin (\sqrt{\lambda} x) \\
\left.\quad-c_{0} \sin \left(\sqrt{\lambda}\left(x-\eta_{0}\right)\right)\right), \quad \eta_{0} \leq x \leq t \leq \eta_{1} ; \\
\left(\sin (\sqrt{\lambda} t)-c_{0} \sin \left(\sqrt{\lambda}\left(t-\eta_{0}\right)\right)\right)(\sin (\sqrt{\lambda}(x-1)) \\
\left.\quad-c_{1} \sin \left(\sqrt{\lambda}\left(x-\eta_{1}\right)\right)\right), \quad \eta_{0} \leq t \leq x \leq \eta_{1} ; \\
\sin (\sqrt{\lambda}(t-1))\left(\sin (\sqrt{\lambda} x)-c_{0} \sin \left(\sqrt{\lambda}\left(x-\eta_{0}\right)\right)\right), \eta_{1} \leq x \leq t \leq 1 ; \\
\sin (\sqrt{\lambda}(x-1))\left(\sin (\sqrt{\lambda} t)-c_{0} \sin \left(\sqrt{\lambda}\left(t-\eta_{0}\right)\right)\right) \\
+c_{1}\left(c_{0} \sin \left(\left(\eta_{0}-\eta_{1}\right) \sqrt{\lambda}\right)+\sin \left(\eta_{1} \sqrt{\lambda}\right)\right) \sin (\sqrt{\lambda}(t-x)), \eta_{1} \leq t \leq x \leq 1 ;
\end{array}\right.
\end{aligned}
$$

and if $\left(P_{0}\right)$ holds, then $\xi^{+}(x, t) \leq 0$, where $\xi^{+}(x, t)$ denotes the Green's function for $\lambda>0$.

Case-2: If $\lambda \in(-\infty, 0)$ then,

$$
\begin{aligned}
& \xi^{-}(x, t)=\frac{1}{D_{\lambda^{-}}} \\
& \times\left\{\begin{array}{l}
\sinh (x \sqrt{|\lambda|})\left(\sinh ((t-1) \sqrt{|\lambda|})-c_{1} \sinh \left(\sqrt{|\lambda|}\left(t-\eta_{1}\right)\right)\right) \\
+c_{0}\left(\sinh \left(\left(\eta_{0}-1\right) \sqrt{|\lambda|}\right)-c_{1} \sinh \left(\left(\eta_{0}-\eta_{1}\right) \sqrt{|\lambda|}\right)\right) \\
\quad \times \sinh (\sqrt{|\lambda|}(t-x)), \quad 0 \leq x \leq t \leq \eta_{0} ; \\
\sinh (t \sqrt{|\lambda|})\left(\sinh ((x-1) \sqrt{|\lambda|})-c_{1} \sinh \left(\sqrt{|\lambda|}\left(x-\eta_{1}\right)\right)\right), \\
\quad 0 \leq t \leq x \leq \eta_{0} ; \\
\left(\sinh ((t-1) \sqrt{|\lambda|})-d \sinh \left(\sqrt{|\lambda|}\left(t-\eta_{1}\right)\right)\right)(\sinh (x \sqrt{|\lambda|})
\end{array}\right. \\
& \left.-c_{0} \sinh \left(\sqrt{|\lambda|}\left(x-\eta_{0}\right)\right)\right), \quad \eta_{0} \leq x \leq t \leq \eta_{1} ; \\
& \left(\sinh (t \sqrt{|\lambda|})-c_{0} \sinh \left(\sqrt{|\lambda|}\left(t-\eta_{0}\right)\right)\right)(\sinh ((x-1) \sqrt{|\lambda|}) \\
& \left.-c_{1} \sinh \left(\sqrt{|\lambda|}\left(x-\eta_{1}\right)\right)\right), \quad \eta_{0} \leq t \leq x \leq \eta_{1} ;
\end{aligned}
$$




$$
\left\{\begin{array}{l}
\sinh ((t-1) \sqrt{|\lambda|})\left(\sinh (x \sqrt{|\lambda|})-c_{0} \sinh \left(\sqrt{|\lambda|}\left(x-\eta_{0}\right)\right)\right), \\
\quad \eta_{1} \leq x \leq t \leq 1 ; \\
\sinh ((x-1) \sqrt{|\lambda|})\left(\sinh (t \sqrt{|\lambda|})-c_{0} \sinh \left(\sqrt{|\lambda|}\left(t-\eta_{0}\right)\right)\right) \\
+c_{1}\left(c_{0} \sinh \left(\left(\eta_{0}-\eta_{1}\right) \sqrt{|\lambda|}\right)+\sinh \left(\eta_{1} \sqrt{|\lambda|}\right)\right) \sinh (\sqrt{|\lambda|}(t-x)), \\
\quad \eta_{1} \leq t \leq x \leq 1 .
\end{array}\right.
$$

and if $\left(N_{0}\right)$ holds, then $\xi^{-}(x, t) \leq 0$, where $\xi^{-}(x, t)$ denotes the Green's function for $\lambda<0$.

Proof. With the help of article $[28,29]$ we can construct the Green's function for the BVPs (3.1), and fix its sign.

Lemma 4. If $\psi(x) \in C^{2}[0,1]$ is any solution of Dirichlet four-point BVPs (2.1), then $\psi(x)$ is given by,

(a). If $\lambda \in I_{0}$, then

$$
\begin{aligned}
\psi(x)= & \frac{1}{D_{\lambda^{+}}} \sqrt{\lambda} A\left(\sin (\sqrt{\lambda} x)-c_{0} \sin \left(\sqrt{\lambda}\left(x-\eta_{0}\right)\right)\right) \\
& -\int_{0}^{1} \xi^{+}(x, t) q(t) d t .
\end{aligned}
$$

(b). If $\lambda \in(-\infty, 0)$, then

$$
\begin{aligned}
\psi(x)= & \frac{1}{D_{\lambda^{-}}} \sqrt{|\lambda|} A\left(\sinh (\sqrt{|\lambda|} x)-c_{0} \sinh \left(\sqrt{|\lambda|}\left(x-\eta_{0}\right)\right)\right) \\
& -\int_{0}^{1} \xi^{-}(x, t) q(t) d t .
\end{aligned}
$$

Proof. $\quad$ For the proof of this lemma we refer [29, lemma 3.2].

Lemma 5. If $\psi(x)$ be a function given in Lemma $4, \delta_{2}:[0,1] \rightarrow[0, \infty)$ such that $\delta_{2}(0)=0$, then we have the following:

(a). If $\left(P_{0}\right)$ is true, $\delta_{1} \in \mathbb{R}^{+}$such that $\left(\lambda-\delta_{1}\right) \leq 0$, and $\delta_{2}^{\prime}(x) \geq 0$, then

$$
\left(\lambda-\delta_{1}\right) \xi^{+}(x, t)+\delta_{2}(x) \operatorname{sign}\left(\psi^{\prime}(x)\right) \frac{\partial \xi^{+}(x, t)}{\partial x} \geq 0, \quad x \neq t, \quad \forall t, x \in[0,1],
$$

where $\frac{\partial \xi^{+}(x, t)}{\partial x}$ is the derivative of $\xi^{+}(x, t)$ with respect to $x$, given by

$$
\begin{aligned}
& \frac{\partial \xi^{+}(x, t)}{\partial x}=\frac{\sqrt{\lambda}}{D_{\lambda^{+}}} \\
& \times\left\{\begin{array}{c}
c_{0}\left(c_{1} \sin \left(\left(\eta_{0}-\eta_{1}\right) \sqrt{\lambda}\right)-\sin \left(\left(\eta_{0}-1\right) \sqrt{\lambda}\right)\right) \cos (\sqrt{\lambda}(t-x)) \\
+\cos (\sqrt{\lambda} x)\left(\sin (\sqrt{\lambda}(t-1))-c_{1} \sin \left(\sqrt{\lambda}\left(t-\eta_{1}\right)\right)\right), \\
0 \leq x \leq t \leq \eta_{0}
\end{array}\right.
\end{aligned}
$$




$$
\left\{\begin{array}{l}
-\sin (\sqrt{\lambda} t)\left(c_{1} \cos \left(\sqrt{\lambda}\left(x-\eta_{1}\right)\right)-\cos (\sqrt{\lambda}(x-1))\right), \\
0 \leq t \leq x \leq \eta_{0} ; \\
-\left(\sin (\sqrt{\lambda}(t-1))-c_{1} \sin \left(\sqrt{\lambda}\left(t-\eta_{1}\right)\right)\right) \\
\quad\left(c_{0} \cos \left(\sqrt{\lambda}\left(x-\eta_{0}\right)\right)-\cos (\sqrt{\lambda} x)\right), \quad \eta_{0} \leq x \leq t \leq \eta_{1} ; \\
\left(\sin (\sqrt{\lambda} t)-c_{0} \sin \left(\sqrt{\lambda}\left(t-\eta_{0}\right)\right)\right)(\cos (\sqrt{\lambda}(x-1)) \\
\left.\quad-c_{1} \cos \left(\sqrt{\lambda}\left(x-\eta_{1}\right)\right)\right), \quad \eta_{0} \leq t \leq x \leq \eta_{1} ; \\
\sin (\sqrt{\lambda}(t-1))\left(\cos (\sqrt{\lambda} x)-c_{0} \cos \left(\sqrt{\lambda}\left(x-\eta_{0}\right)\right)\right), \eta_{1} \leq x \leq t \leq 1 ; \\
\cos (\sqrt{\lambda}(x-1))\left(\sin (\sqrt{\lambda} t)-c_{0} \sin \left(\sqrt{\lambda}\left(t-\eta_{0}\right)\right)\right) \\
-c_{1}\left(c_{0} \sin \left(\left(\eta_{0}-\eta_{1}\right) \sqrt{\lambda}\right)+\sin \left(\eta_{1} \sqrt{\lambda}\right)\right) \cos (\sqrt{\lambda}(t-x)), \\
\eta_{1} \leq t \leq x \leq 1 .
\end{array}\right.
$$

(b). If $\left(N_{0}\right)$ is true and $\delta_{1} \in \mathbb{R}^{+}$such that $\left(\lambda+\delta_{1}\right) \leq 0$, then

$$
\left(\lambda+\delta_{1}\right) \xi^{-}(x, t)+\delta_{2}(x) \operatorname{sign}\left(\psi^{\prime}(x)\right) \frac{\partial \xi^{-}(x, t)}{\partial x} \geq 0, x \neq t, \forall t, x \in[0,1],
$$

where $\frac{\partial \xi^{-}(x, t)}{\partial x}$ is the derivative of $\xi^{-}(x, t)$ with respect to $x$, which can be obtained from Equation (3.3).

Proof. (a.) The result can be easily obtained by using (3.2) and (3.6), Lemma 1, and $\left(P_{0}\right)$. Let us first prove that,

$$
\left(\lambda-\delta_{1}\right) \xi^{+}(x, t)+\delta_{2}(x) \frac{\partial \xi^{+}(x, t)}{\partial x} \geq 0 .
$$

Consider the case $0 \leq x \leq t \leq \eta_{0}$, from (3.2) and (3.6), we symbolize

$$
\begin{aligned}
& \xi_{1}^{+}(x, t)=\frac{1}{D_{\lambda^{+}}}\left[\sin (\sqrt{\lambda} x)\left(\sin (\sqrt{\lambda}(t-1))-c_{1} \sin \left(\sqrt{\lambda}\left(t-\eta_{1}\right)\right)\right)\right. \\
& \left.\quad+c_{0}\left(\sin \left(\left(\eta_{0}-1\right) \sqrt{\lambda}\right)-c_{1} \sin \left(\left(\eta_{0}-\eta_{1}\right) \sqrt{\lambda}\right)\right) \sin (\sqrt{\lambda}(t-x))\right], \\
& \frac{\partial \xi_{1}^{+}(x, t)}{\partial x}=\frac{\sqrt{\lambda}}{D_{\lambda^{+}}}\left[c_{0}\left(c_{1} \sin \left(\left(\eta_{0}-\eta_{1}\right) \sqrt{\lambda}\right)-\sin \left(\left(\eta_{0}-1\right) \sqrt{\lambda}\right)\right)\right. \\
& \left.+\cos (\sqrt{\lambda}(t-x)) \cos (\sqrt{\lambda} x)\left(\sin (\sqrt{\lambda}(t-1))-c_{1} \sin \left(\sqrt{\lambda}\left(t-\eta_{1}\right)\right)\right)\right] .
\end{aligned}
$$

Then, putting in (3.7), we get

$$
\begin{aligned}
& \left(\lambda-\delta_{1}\right) \xi_{1}^{+}(x, t)+\delta_{2}(x) \frac{\partial \xi_{1}^{+}(x, t)}{\partial x} \\
& =\frac{1}{D_{\lambda^{+}}}\left[H_{1}(t)\left(\left(\lambda-\delta_{1}\right) \sin (\sqrt{\lambda} x)\right)+\delta_{2}(x) \sqrt{\lambda} \cos (\sqrt{\lambda} x)\right. \\
& \left.\quad+c_{0} K_{1}\left(\left(\lambda-\delta_{1}\right) \sin (\sqrt{\lambda}(t-x))-\delta_{2}(x) \sqrt{\lambda} \cos (\sqrt{\lambda}(t-x))\right)\right] \geq 0,
\end{aligned}
$$


where

$$
\begin{aligned}
& H_{1}(t)=\sin (\sqrt{\lambda}(t-1))-c_{1} \sin \left(\sqrt{\lambda}\left(t-\eta_{1}\right)\right) \\
& K_{1}=\sin \left(\left(\eta_{0}-1\right) \sqrt{\lambda}\right)-c_{1} \sin \left(\left(\eta_{0}-\eta_{1}\right) \sqrt{\lambda}\right)
\end{aligned}
$$

are non-positive under $\left(P_{0}\right)$. By applying Lemma 1(a) it can be proved that, $\left(\lambda-\delta_{1}\right) \sin (\sqrt{\lambda}(t-x))-\delta_{2}(x) \sqrt{\lambda} \cos (\sqrt{\lambda}(t-x)) \leq 0$. Hence proved. Similarly, for rest of the cases, we can prove the result easily by using $\left(P_{0}\right)$ and Lemma 1. Proof of $(b)$ follows similarly.

Proposition 1. Maximum principle: If $q(x) \in C[0,1]$ is a non negative function and $A \geq 0$ is any constant then the following conditions hold,

(a). If $\left(P_{0}\right)$ is true, then the solution $\psi(x) \in C^{2}[0,1]$ of BVPs $(2.1)$, given by (3.4), is non-negative.

(b). If $\left(N_{0}\right)$ is true, then the solution $\psi(x) \in C^{2}[0,1]$ of BVPs (2.1), given by (3.5), is non-negative.

Proof. (a) Given that $q(x) \geq 0, A \geq 0$, and $\left(P_{0}\right)$ is satisfied. From Case-1 of Lemma 3, we have $\xi^{+}(x, t) \leq 0$. Now to prove that $\psi(x) \geq 0, \forall x \in[0,1]$ it is sufficient to prove $\sin (\sqrt{\lambda} x)-c_{0} \sin \left(\sqrt{\lambda}\left(x-\eta_{0}\right)\right) \geq 0$. Therefore we have,

$$
\begin{aligned}
& \sin (\sqrt{\lambda} x)-c_{0} \sin \left(\sqrt{\lambda}\left(x-\eta_{0}\right)\right) \\
& \quad=\sin (\sqrt{\lambda} x)\left(1-c_{0} \cos \left(\sqrt{\lambda} \eta_{0}\right)\right)+c_{0} \sin \left(\sqrt{\lambda} \eta_{0}\right) \cos (\sqrt{\lambda} x) \geq 0
\end{aligned}
$$

as $1-c_{0} \cos \left(\sqrt{\lambda} \eta_{0}\right) \geq 0$ for $c_{0} \in(0,1)$. Hence proved. Similar process is applied to prove $(b)$.

\section{The nonlinear problem}

Here we define lower solution $l(x)$, upper solution $u(x)$, construct iterative sequences with initial iterates of L-U solutions, and prove the existence result. Nagumo conditions are used to show the bound on the derivative of the solution. We have also assumed some conditions on nonlinear term $F\left(x, \psi, \psi^{\prime}\right)$ based on $l(x)$ and $u(x)$. Here the Lipschitz constant $\delta_{2}(x) \geq 0$, with respect to $\psi^{\prime}$, is a function of $x$, if we do not assume this then it is not easy to generate the monotonic sequences.

DEFINITION 1. Lower solution and upper solution: $l(x) \in C^{2}([0,1])$, is called lower solution of (1.1) if it satisfy,

$$
l^{\prime \prime}(x)+F\left(x, l(x), l^{\prime}(x)\right) \geq 0, \operatorname{BCs}(0)=0, \quad \operatorname{BCs}(1) \leq 0, \quad 0<x<1,
$$

where $\operatorname{BCs}(i), i=0,1$, is defined in (1.1). Similarly, a function $u(x) \in$ $C^{2}([0,1])$, is called an upper solution of (1.1) if it satisfy, (4.1) with reverse inequality. 
Let $\left(l_{m}(x)\right)_{m},\left(u_{m}(x)\right)_{m} \in C^{2}([0,1]), m \in \mathbb{N}$, are two sequences defined as follows:

$$
\begin{array}{r}
-l_{m+1}^{\prime \prime}(x)-\lambda l_{m+1}(x)=F\left(x, l_{m}(x), l_{m}^{\prime}(x)\right)-\lambda l_{m}(x), \\
l_{m+1}(i)-c_{i} l_{m+1}\left(\eta_{i}\right)=0, \quad i=0,1, \\
-u_{m+1}^{\prime \prime}(x)-\lambda u_{m+1}(x)=F\left(x, u_{m}(x), u_{m}^{\prime}(x)\right)-\lambda u_{m}(x), \\
u_{m+1}(i)-c_{i} u_{m+1}\left(\eta_{i}\right)=0, \quad i=0,1 .
\end{array}
$$

If $m=0$, define $l_{0}=l$ and $u_{0}=u$, where $l$ and $u$ are L-U solutions of Equation (1.1), respectively.

If $\lambda \in I_{0} \cup(-\infty, 0)$ then assume that the following hypotheses hold.

$\left(M_{1}\right)$ : There exist L-U solutions $l(x), u(x) \in C^{2}([0,1])$ such that $l(x) \leq$ $u(x), \forall 0 \leq x \leq 1$.

$\left(M_{2}\right):$ Let $F(x, v, z): E \rightarrow \mathbb{R}$ is continuous on $E$, where $E:=\{(x, v, z) \in$ $\Omega: l(x) \leq v(x) \leq u(x)\}$ and $\Omega=[0,1] \times \mathbb{R}^{2}$.

$\left(M_{3}\right)$ : There exists a constant $\delta_{2}(x) \geq 0$ such that

$$
\left|F\left(x, v, z_{2}\right)-F\left(x, v, z_{1}\right)\right| \leq \delta_{2}(x)\left|\left(z_{2}-z_{1}\right)\right|, \quad \forall\left(x, v, z_{i}\right) \in E, \quad i=1,2 .
$$

$\left(M_{4}\right)$ : Suppose $\vartheta \in C\left(\mathbb{R}^{+}, \mathbb{R}^{+}\right)$such that $|F(x, v, z)| \leq \vartheta(|z|)$, which satisfies,

$$
\max _{x \in I} u(x)-\min _{x \in I} l(x)<\int_{\Upsilon}^{\infty} \frac{\mu d \mu}{\vartheta(\mu)}
$$

where $C\left(\mathbb{R}^{+}, \mathbb{R}^{+}\right)$denotes set of all continuous functions $\vartheta: \mathbb{R}^{+} \rightarrow \mathbb{R}^{+}$and $\Upsilon=2 \max \left\{\sup _{x \in I}|u(x)|, \sup _{x \in I}|l(x)|\right\}$.

Case-1: If $\lambda \in I_{0}$, then suppose $\left(P_{1}\right):$ There exists a constant $\delta_{1} \geq 0$ such that $v_{1} \leq v_{2} \Rightarrow F\left(x, v_{2}, z\right)-F\left(x, v_{1}, z\right) \geq \delta_{1}\left(v_{2}-v_{1}\right) ; \forall\left(x, v_{i}, z\right) \in E$, where $i=1,2$.

Case-2: If $\lambda \in(-\infty, 0)$, then suppose $\left(N_{1}\right)$ : There exists a constant $\delta_{1} \geq 0$ such that

$$
v_{1} \leq v_{2} \Rightarrow F\left(x, v_{2}, z\right)-F\left(x, v_{1}, z\right) \geq-\delta_{1}\left(v_{2}-v_{1}\right) ; \quad \forall\left(x, v_{i}, z\right) \in E, i=1,2 .
$$

\subsection{The existence theorem}

In this subsection, we prove monotonic behavior of L- $\mathrm{U}$ solution in well ordered case and existence result. This section is divided into two subsections, in first we see the results for $\lambda \in I_{0}$ and in other we see the results for $\lambda \in(-\infty, 0)$.

Lemma 6. If function $F\left(x, \psi, \psi^{\prime}\right)$ satisfies $\left(M_{4}\right)$ then there exists a positive real number $M$ such that $\left\|\psi^{\prime}\right\|_{\infty} \leq M$, where $\psi$ is a solution of

$$
\psi^{\prime \prime}+F\left(x, \psi, \psi^{\prime}\right) \leq 0, \quad B C s(0)=0, \quad B C s(1) \geq 0, \quad 0<x<1,
$$

such that $\psi \in[l(x), u(x)]$, where $l(x)$ and $u(x)$ are $L$-U solutions of (1.1), respectively. 
Proof. We prove lemma for the following three cases.

Case-1: If $\psi^{\prime}>0, \forall x \in(0,1)$, i.e., $\psi$ is strictly increasing in $(0,1)$. Then there exists a constant $\zeta$ such that $\psi^{\prime}(\zeta)=\psi(1)-\psi(0)$. Hence $\left|\psi^{\prime}(\zeta)\right| \leq \Upsilon$, where $\Upsilon=2 \max \left\{\sup _{x \in[0,1]}|u(x)|, \sup _{x \in[0,1]}|l(x)|\right\}$. Now we have,

$$
\int_{\Upsilon}^{u^{\prime}(x)} \frac{\mu d \mu}{\vartheta(|\mu|)} \leq \int_{u^{\prime}(\zeta)}^{u^{\prime}(x)} \frac{\mu d \mu}{\vartheta(|\mu|)} .
$$

From condition $\left(M_{4}\right)$ and Equation (4.4), we have $u^{\prime \prime}(x) \leq \vartheta\left(\left|u^{\prime}\right|\right)$. Now multiplying by $u^{\prime}(x)$ in both sides of this inequality and then integrating from $\zeta$ to $x$, we get

$$
\int_{\zeta}^{x} \frac{u^{\prime \prime}(x) u^{\prime}(x) d x}{\vartheta\left(\left|u^{\prime}\right|\right)} \leq \int_{\zeta}^{x} u^{\prime}(x) d x
$$

Let us put $u^{\prime}(x)=\mu$, then there exist a constant $M$ satisfying,

$$
\int_{u^{\prime}(\zeta)}^{u^{\prime}(x)} \frac{\mu d \mu}{\vartheta(|\mu|)} \leq \max _{x \in I} u(x)-\min _{x \in I} l(x) \leq \int_{\Upsilon}^{M} \frac{\mu d \mu}{\vartheta(|\mu|)}, \quad\left\{\operatorname{using}\left(M_{4}\right)\right\} .
$$

Hence applying Equation (4.5) in Equation (4.6), we have

$$
\int_{\Upsilon}^{u^{\prime}(x)} \frac{\mu d \mu}{\vartheta(|\mu|)} \leq \int_{0}^{M} \frac{\mu d \mu}{\vartheta(|\mu|)}, \quad \Rightarrow u^{\prime}(x) \leq M .
$$

This completes the proof for Case-1.

Case-2: If $\psi^{\prime}<0$, i.e., $\psi$ is strictly decreasing in $(0,1)$. We can prove the result as in Case-1.

Case-3: If $\psi$ is neither monotonically increasing nor monotonically decreasing through out the interval then we consider two subcases.

Subcase-1: We consider the sub interval $\left(x_{0}, x\right] \subset(0,1)$ such that $\psi^{\prime}\left(x_{0}\right)=0$, and $\psi^{\prime}(x)>0$ for $x_{0}<x$. To prove the result we follow proof of Case- 1 .

Subcase-2: In this subcase, we consider the sub interval $\left[x, x_{0}\right) \subset(0,1)$ such that $\psi^{\prime}\left(x_{0}\right)=0$, and $\psi^{\prime}(x)<0$ for $x_{0}>x$, and proceed similarly.

Lemma 7. If function $F\left(x, \psi, \psi^{\prime}\right)$ satisfies $\left(M_{4}\right)$ then there exists a positive real number $M$ such that $\left\|\psi^{\prime}\right\|_{\infty} \leq M$, where $\psi$ is a solution of

$$
\psi^{\prime \prime}+F\left(x, \psi, \psi^{\prime}\right) \geq 0, \quad B C s(0)=0, \quad B C s(1) \leq 0, \quad 0<x<1,
$$

such that $\psi \in[l(x), u(x)]$, where $l(x)$ and $u(x)$ are $L$ - $U$ solution of (1.1), respectively.

Proof. We can prove this result similarly as in Lemma 6.

Lemma 8. If $\lambda \neq 0$. Suppose $\left(l_{m}(x)\right)_{m},\left(u_{m}(x)\right)_{m} \in C^{2}([0,1])$, defined in (4.2)-(4.3), are $L$ - $U$ solution of $(3.1)$, then $l_{m+1}(x)-l_{m}(x) \geq 0$, and $u_{m+1}(x)-$ $u_{m}(x) \leq 0, \forall m \in \mathbb{N}, \forall x \in[0,1]$.

Proof. See Lemma 5.1 of [29]. 


\subsection{1 $\lambda \in I_{0}$}

Proposition 2. If $\lambda \in I_{0}, \delta_{1} \in \mathbb{R}^{+}$, and $\delta_{2}:[0,1] \rightarrow[0, \infty)$ such that $\left(\lambda-\delta_{1}\right) \leq$ $0, \delta_{2}(0)=0, \delta_{2}^{\prime}(x) \geq 0$, and lemma 1 holds. Also if $\left(P_{0}\right),\left(P_{1}\right)$, and $\left(M_{1}\right)-\left(M_{3}\right)$ hold, then $\left(l_{m}(x)\right)_{m},\left(u_{m}(x)\right)_{m}$, defined in (4.2)-(4.3), are such that $\forall m \in \mathbb{N}$ and $x \in[0,1]$,

1. $\left(l_{m}(x)\right)_{m}$ is lower solution of $(1.1)$ and $l_{m+1}(x)-l_{m}(x) \geq 0$,

2. $\left(u_{m}(x)\right)_{m}$ is upper solution of (1.1) and $u_{m+1}(x)-u_{m}(x) \leq 0$.

Proof. Proof of (1.) To prove that $\left(l_{m}(x)\right)_{m}$ is lower solution of (1.1) such that $l_{m+1}(x)-l_{m}(x) \geq 0$, we use principle of mathematical induction. For $m=0$, we have $l_{0}(x)=l(x)$ is lower solution of $(1.1)$, hence by Lemma 8 , $l_{1}(x)-l_{0}(x) \geq 0$. Let the result is true for $m-1$, i.e., $l_{m-1}(x)$, is lower solution of $(1.1)$ and $l_{m}(x)-l_{m-1}(x) \geq 0$. Now to prove the claim from $\left(M_{3}\right),\left(P_{1}\right)$, and Equation (4.2), we have,

$$
-l_{m}^{\prime \prime}-F\left(x, l_{m}, l_{m}^{\prime}\right)=\lambda\left(l_{m}-l_{m-1}\right)+\delta_{1}\left(l_{m-1}-l_{m}\right)+\delta_{2}\left|l_{m-1}^{\prime}-l_{m}^{\prime}\right| .
$$

Now let, $\psi(x)=l_{m}(x)-l_{m-1}(x)$. Then we arrive at,

$$
-l_{m}^{\prime \prime}(x)-F\left(x, l_{m}(x), l_{m}^{\prime}(x)\right)=\left(\lambda-\delta_{1}\right) \psi(x)+\delta_{2}(x) \operatorname{sign}\left(\psi^{\prime}(x)\right)\left|\left(\psi^{\prime}(x)\right)\right| .
$$

Since, $l_{m-1}(x)$ is lower solution of (1.1). Therefore, $\psi(x)$ satisfies,

$$
\begin{aligned}
& -\psi^{\prime \prime}-\lambda \psi=l_{m-1}^{\prime \prime}+F\left(x, l_{m-1}, l_{m-1}^{\prime}\right) \geq 0, \\
& B C s(0)=0, \quad B C s(1) \geq 0 .
\end{aligned}
$$

Hence, we conclude that $\psi(x)$ is a solution of (2.1), and it can be written in the form of (3.4). So, to prove $l_{m}(x)$ is lower solution of (1.1) it is necessary to prove $\left(\lambda-\delta_{1}\right) \psi(x)+\delta_{2} \operatorname{sign}\left(\psi^{\prime}(x)\right)\left|\left(\psi^{\prime}(x)\right)\right| \leq 0$, which can be obtained by using Lemmas 1 and 5. Proof for (2.) is similar. Hence the result.

Proposition 3. If $\lambda \in I_{0}, \delta_{1} \in \mathbb{R}^{+}$, and $\delta_{2}:[0,1] \rightarrow[0, \infty)$ such that $\left(\lambda-\delta_{1}\right) \leq$ $0, \delta_{2}(0)=0, \delta_{2}^{\prime}(x) \geq 0$, and lemma 1 holds. Also if $\left(P_{0}\right),\left(P_{1}\right)$, and $\left(M_{1}\right)-\left(M_{3}\right)$ hold, and

$$
F\left(x, u(x), u^{\prime}(x)\right)-F\left(x, l(x), l^{\prime}(x)\right)-\lambda(u(x)-l(x)) \geq 0,
$$

then $\left(l_{m}(x)\right)_{m},\left(u_{m}(x)\right)_{m}$, defined in (4.2)-(4.3) are such that $\forall m \in \mathbb{N}$ and $x \in[0,1], l_{m}(x)-u_{m}(x) \leq 0$.

Proof. Proof of this proposition can be shown with the help of proposition 5 in $[29]$.

Theorem 1. If $\lambda \in I_{0}, \delta_{1} \in \mathbb{R}^{+}$, and $\delta_{2}:[0,1] \rightarrow[0, \infty)$ such that $\left(\lambda-\delta_{1}\right) \leq 0$, $\delta_{2}(0)=0, \delta_{2}^{\prime}(x) \geq 0$, and Lemma 1 holds. Also if $\left(P_{0}\right),\left(P_{1}\right)$, and $\left(M_{1}\right)-\left(M_{3}\right)$ hold, and

$$
F\left(x, u(x), u^{\prime}(x)\right)-F\left(x, l(x), l^{\prime}(x)\right)-\lambda(u(x)-l(x)) \geq 0,
$$


then the sequences $\left(l_{m}(x)\right)_{m},\left(u_{m}(x)\right)_{m}$ monotonically converges in $C^{1}[0,1]$ to the solution $l^{*}(x)$ and $u^{*}(x)$ of the nonlinear Equation (1.1), such that $l(x) \leq$ $l^{*}(x) \leq u^{*}(x) \leq u(x)$.

Proof. Using Propositions 2 and 3, we obtain that the sequences $\left(l_{m}(x)\right)_{m}$ and $\left(u_{m}(x)\right)_{m}$ such that,

$$
l(x)=l_{0}(x) \leq l_{1}(x) \ldots \leq l_{m}(x) \ldots \leq u_{m}(x) \leq \ldots \leq u_{1}(x) \leq u_{0}(x)=u(x) .
$$

From (4.7), we can conclude that $\left(l_{m}(x)\right)_{m}$ is monotonically increasing and $\left(u_{m}(x)\right)_{m}$ is monotonically decreasing. Also $\left(l_{m}(x)\right)_{m}$ and $\left(u_{m}(x)\right)_{m}$ are bounded. Therefore by monotone convergence theorem, $\left(l_{m}(x)\right)_{m}$ converges to its supremum, say $l^{*}(x)$; and $\left(u_{m}(x)\right)_{m}$ converges to its infimum, say $u^{*}(x)$. Hence we can write,

$$
\lim _{m \rightarrow \infty} l_{m}(x)=l^{*}(x) \text { and } \lim _{m \rightarrow \infty} u_{m}(x)=u^{*}(x),
$$

such that $l_{m}(x) \leq l^{*}(x) \leq u^{*}(x) \leq u_{m}(x), \forall x \in[0,1]$. From Equations (4.2)(4.3), (4.7) and Lemmas 6-7, we can conclude that $\left(l_{m}\right)_{m}$ and $\left(u_{m}\right)_{m}$ are uniformly bounded and equicontinuous in $C^{1}[0,1]$. Let $\left(l_{m_{i}}\right)_{i}$ and $\left(u_{m_{i}}\right)_{i}$ are any subsequences of $\left(l_{m}\right)_{m}$ and $\left(u_{m}\right)_{m}$ respectively, then $\left(l_{m_{i}}\right)_{i}$ and $\left(u_{m_{i}}\right)_{i}$ are also uniformly bounded and equicontinuous in $C^{1}[0,1]$. With the help of Arzela Ascoli theorem, we conclude that these subsequences $\left(l_{m_{i}}\right)_{i}$ and $\left(u_{m_{i}}\right)_{i}$ also contain uniformly convergent subsequences in $C^{1}[0,1]$. Since $\left(l_{m}\right)_{m}$ and $\left(u_{m}\right)_{m}$ are monotone. And by uniqueness of limit we have, $l_{m}(x) \rightarrow l^{*}(x)$ in $C^{1}[0,1]$ uniformly and $u_{m}(x) \rightarrow u^{*}(x)$ in $C^{1}[0,1]$ uniformly.

The solution of (4.2)-(4.3) can be written by Equation (3.4) of Lemma 4 and taking limit on both sides we can conclude existence of solution of NLB$\operatorname{VPs}(1.1)$.

\subsection{2 $\lambda \in(-\infty, 0)$}

We skip the proof of this section, as all the proofs are similar to the proof of the above subsection, for $\lambda \in I_{0}$.

Proposition 4. If $\lambda \in(-\infty, 0), \delta_{1} \in \mathbb{R}^{+}$, and $\delta_{2}:[0,1] \rightarrow[0, \infty)$ such that $\left(\lambda+\delta_{1}\right) \leq 0, \delta_{2}(0)=0$ and Lemma 2 holds. Also if $\left(N_{0}\right),\left(N_{1}\right)$, and $\left(M_{1}\right)-\left(M_{3}\right)$ hold, then $\left(l_{m}(x)\right)_{m},\left(u_{m}(x)\right)_{m}$, defined in (4.2)-(4.3), are such that $\forall m \in \mathbb{N}$ and $x \in[0,1]$,

1. $\left(l_{m}(x)\right)_{m}$ is lower solution of $(1.1)$ and $l_{m+1}(x)-l_{m}(x) \geq 0$.

2. $\left(u_{m}(x)\right)_{m}$ is upper solution of (1.1) and $u_{m+1}(x)-u_{m}(x) \leq 0$.

Proposition 5. If $\lambda \in(-\infty, 0), \delta_{1} \in \mathbb{R}^{+}$, and $\delta_{2}:[0,1] \rightarrow[0, \infty)$ such that $\left(\lambda+\delta_{1}\right) \leq 0, \delta_{2}(0)=0$ and Lemma 2 holds. Also if $\left(N_{0}\right),\left(N_{1}\right)$, and $\left(M_{1}\right)-\left(M_{3}\right)$ hold, and

$$
F\left(x, u(x), u^{\prime}(x)\right)-F\left(x, l(x), l^{\prime}(x)\right)-\lambda(u(x)-l(x)) \geq 0,
$$

then $\left(l_{m}(x)\right)_{m},\left(u_{m}(x)\right)_{m}$, defined in (4.2)-(4.3) are such that $\forall m \in \mathbb{N}$ and $x \in[0,1], l_{m}(x)-u_{m}(x) \leq 0$. 
Theorem 2. If $\lambda \in(-\infty, 0)$ and $\delta_{1} \in \mathbb{R}^{+}$such that $\left(\lambda+\delta_{1}\right) \leq 0$. Let $\delta_{2}$ : $[0,1] \rightarrow[0, \infty)$ such that $\delta_{2}(0)=0$, and Lemma 2 holds. Also if $\left(N_{0}\right),\left(N_{1}\right)$, and $\left(M_{1}\right)-\left(M_{3}\right)$ hold, and

$$
F\left(x, u(x), u^{\prime}(x)\right)-F\left(x, l(x), l^{\prime}(x)\right)-\lambda(u(x)-l(x)) \geq 0,
$$

then the sequences $\left(l_{m}(x)\right)_{m},\left(u_{m}(x)\right)_{m}$ monotonically converge in $C^{1}[0,1]$ to the solution $l^{*}(x)$ and $u^{*}(x)$ of the nonlinear Equation (1.1), such that $l(x) \leq$ $l^{*}(x) \leq u^{*}(x) \leq u(x)$.

\section{Numerical verifications}

In this section, we verify our theoretical results by three examples. First two examples are for $\lambda \in I_{0}$ and the last example is for $\lambda \in(-\infty, 0)$. We have used Mathematica 11.3 version for plotting.

Example 1. Consider the four point NLBVPs,

$$
\begin{aligned}
-\psi^{\prime \prime}(x) & =\frac{9 e^{\psi}}{22}-\frac{3}{28} x e^{\psi^{\prime}}, \quad 0<x<1, \\
\operatorname{BCs}(i) & =\psi(i)-c_{i} \psi\left(\eta_{i}\right)=0, \quad i=0,1,
\end{aligned}
$$

where $F=\frac{9 e^{\psi}}{22}-\frac{3}{28} x e^{\psi^{\prime}}$ and $c_{0}=0.2, c_{1}=1, \eta_{0}=0.6$ and $\eta_{1}=0.8$. Taking initial lower solution $l_{0}(x)=\frac{1}{5} x^{2}-0.5 x-0.057$ and initial upper solution $u_{0}(x)=2\left(\frac{-1}{5} x^{2}+0.5 x+0.057\right)$ such that $l_{0}(x) \leq u_{0}(x)$. The Lipschitz constants are $\delta_{1}=0.28627$ and $\delta_{2}=\frac{3 x e^{M}}{28}$, where $M=0.479061583$. We obtain $\vartheta(|z|)=0.83542235+\frac{3 e^{(|z|)}}{28}$ by using assumption $\left(M_{4}\right)$. Hence we obtain that the sequences of $\mathrm{L}-\mathrm{U}$ solutions converge to the solution of (5.1) for $\lambda \leq 0.113292817$. This value of $\lambda$ is obtained by using inequality

$$
\lambda \leq \min \left(\delta_{1},\left(\delta_{1}-\sup _{x \in[0,1]} \delta_{2}^{\prime}(x)\right), \frac{F\left(x, u, u^{\prime}\right)-F\left(x, l, l^{\prime}\right)}{(u-l)}\right) .
$$

We have plotted the graph of the conditions defined in $\left(P_{0}\right)$. Also, in Figures $1-$ 5 we have shown graphically that the proposed method works and the sequences of L-U solutions monotonically converge to the solution of (5.1).

Example 2. Consider the four-point NLBVPs,

$$
\begin{aligned}
-\psi^{\prime \prime}(x) & =\frac{1}{20}\left(\frac{1}{14} x^{2} e^{\psi}+\frac{19}{2} e^{\psi^{\prime}}\right), \quad 0<x<1, \\
\operatorname{BCs}(i) & =\psi(i)-c_{i} \psi\left(\eta_{i}\right)=0, \quad i=0,1,
\end{aligned}
$$

where $c_{0}=0.2, c_{1}=1.2, \eta_{0}=0.4$ and $\eta_{1}=0.5$. Initial iterations of L-U solutions are $l_{0}(x)=0$ and $u_{0}(x)=\frac{1}{4}\left(-\frac{3}{2} x^{2}+3 x+0.24\right)$ which are in well ordered case. Again $\delta_{1}=0.475$ and $\delta_{2}(x)=\frac{x^{2} e^{M}}{280}$, where $M=0.05574175147$. Here $\vartheta(|z|)=\frac{19}{40}+\frac{e^{(|z|)}}{280}$. We obtain the sequences of L-U solutions converge in the range of $\lambda \in(0,0.4674] \subset I_{0}$. Below in Figures $6-11$, we have shown convergence of monotone iterative sequences graphically. 


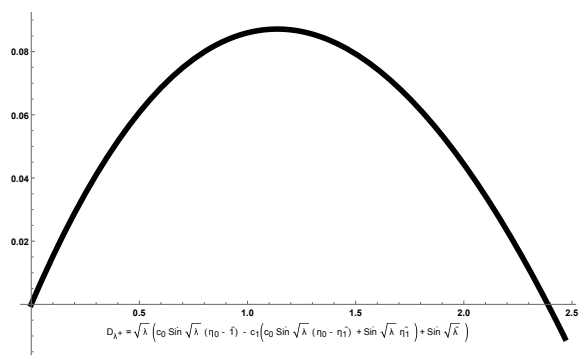

Figure 1. $D_{\lambda}^{+}>0$.

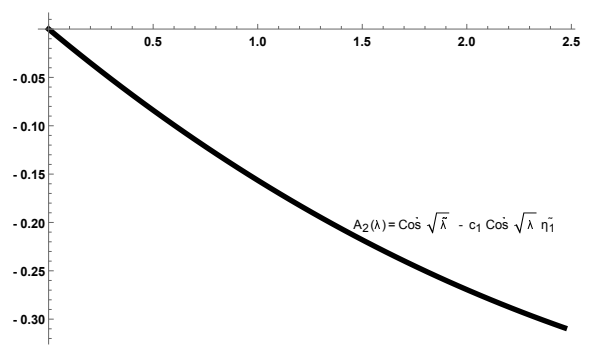

Figure 3. $A_{2}(\lambda) \leq 0$.

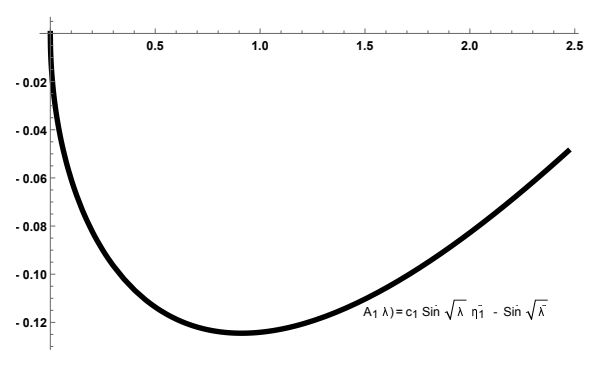

Figure 2. $A_{1}(\lambda)<0$.

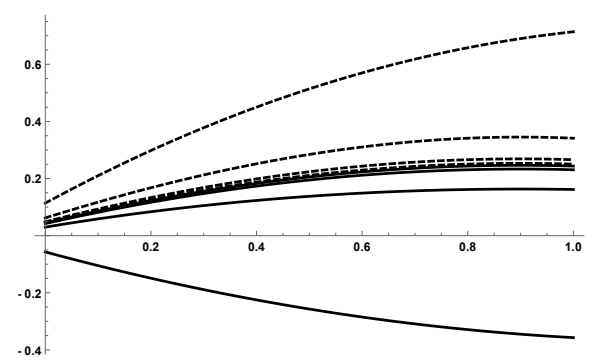

Figure 4. $\lambda=0.05, m=4$.

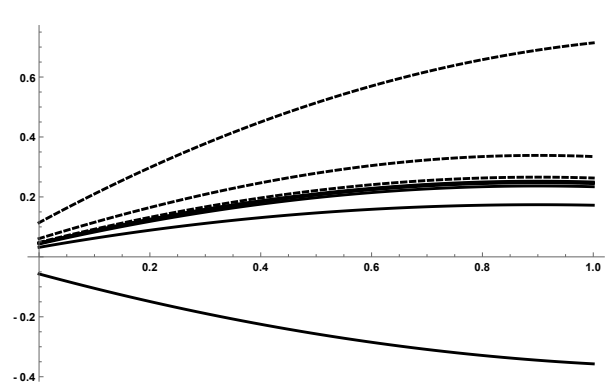

Figure 5. $\lambda=0.1, m=5$.

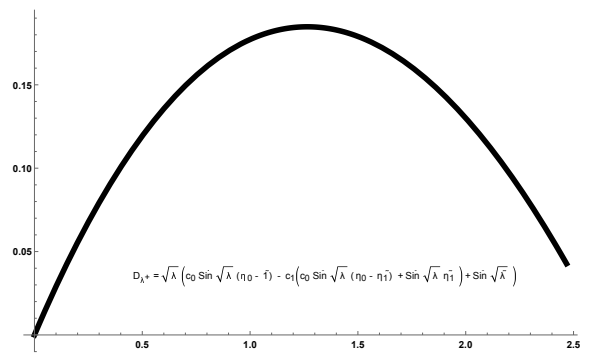

Figure 6. $D_{\lambda}^{+}>0$.

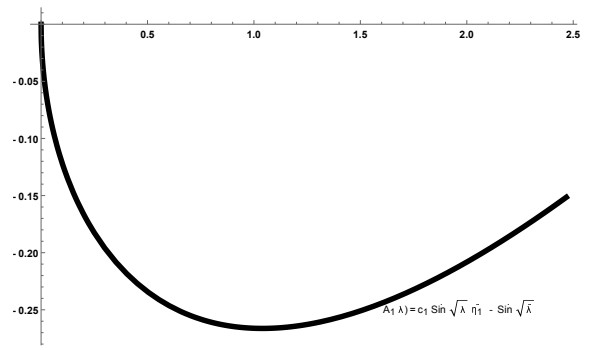

Figure 7. $A_{1}(\lambda)<0$. 


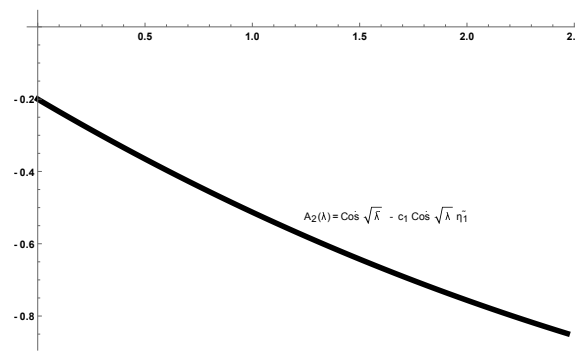

Figure 8. $A_{2}(\lambda) \leq 0$.

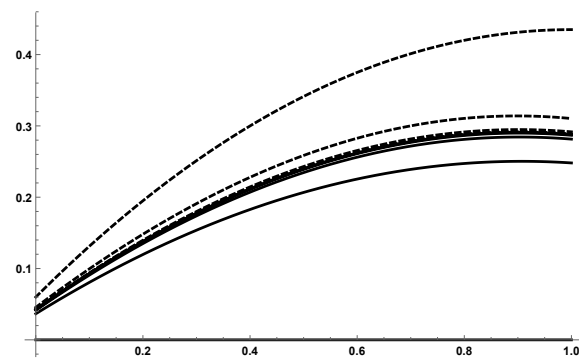

Figure 10. $\lambda=0.2, m=6$.

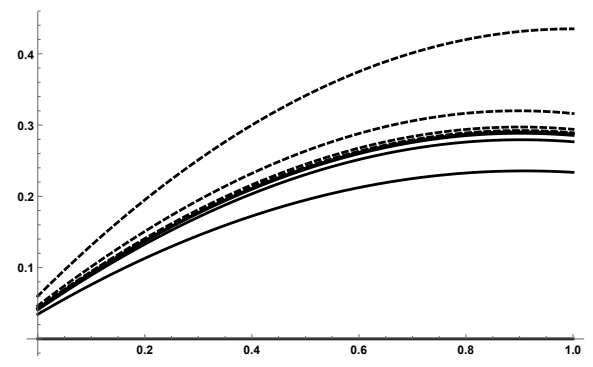

Figure 9. $\lambda=0.05, m=5$.

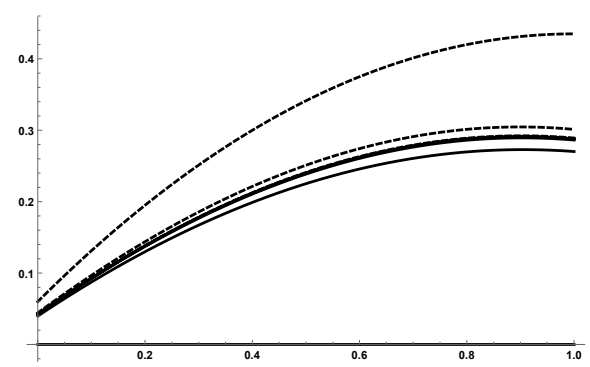

Figure 11. $\lambda=0.4, m=5$.

Example 3. Consider the four point NLBVPs,

$$
\begin{aligned}
-\psi^{\prime \prime}(x) & =\frac{1}{49}\left(e^{\psi}+{\psi^{\prime}}^{3}-\frac{\sin (x)}{8}\right), \\
\mathrm{BC}(i) & =\psi(i)-c_{i} \psi\left(\eta_{i}\right)=0, \quad i=0,1,
\end{aligned}
$$

where $c_{0}=0.2, c_{1}=0.9, \eta_{0}=0.4, \eta_{1}=0.5, l_{0}(x)=\frac{3}{2} x^{2}-3 x-0.24$ and $u_{0}(x)=-\frac{3}{2} x^{2}+3 x+0.24$ such that $l_{0}(x) \leq u_{0}(x)$. For $\lambda<0$ we obtain Lipschitz constant $\delta_{1}=0.262263$ and $\delta_{2}(x)=\frac{x\left(x^{3}+2\right) e^{M}}{49}$, where $M=0.6527$. By using Nagumo condition given by $\left(M_{4}\right)$, we have $\vartheta(|z|)=\frac{3}{49}\left(1.865+e^{|z|}\right)$. We obtain the value of $\lambda$ as,

$$
\begin{aligned}
\lambda & \leq \min \left(-\delta_{1},-\sup \left(\left(\delta_{1}+\delta_{2}\right)+\frac{1}{2} \delta_{2}\left(\delta_{2}+\sqrt{\delta_{2}^{2}+4\left(\delta_{1}+\delta_{2}\right)}\right)\right)\right) \\
& =-0.587610142 .
\end{aligned}
$$

Hence the sequences of L-U solutions converge monotonically in the interval $\lambda \in(-a,-0.587610142) \subset(-\infty, 0)$, where $a$ can be obtained from figure 14. For the different values of $\lambda$ and $m$ graphs (see Figures 12-17) are shown below. We can also see that all the assumption taken in $\left(N_{0}\right)$ are satisfied for the specific region for $\lambda \in(-\infty, 0)$. 


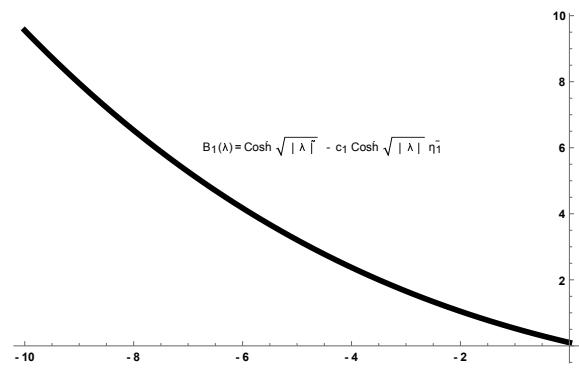

Figure 12. $B_{1}(\lambda) \geq 0$.

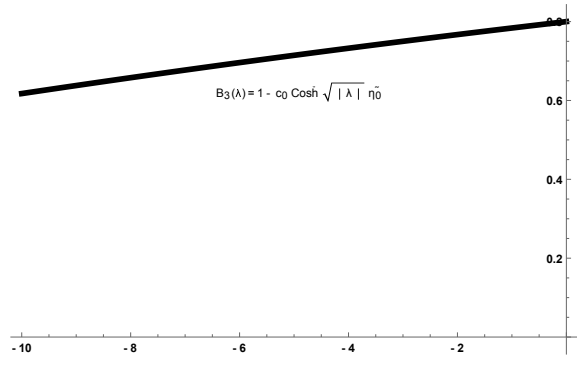

Figure 14. $B_{3}(\lambda)>0$.

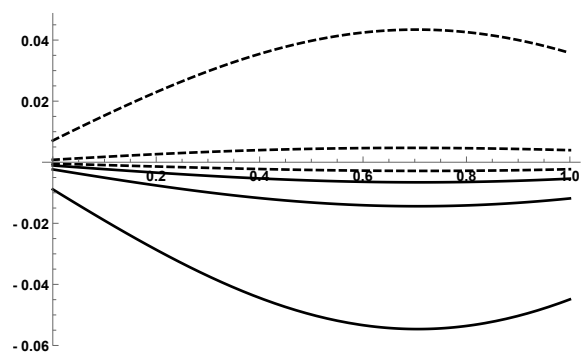

Figure 16. $\lambda=-1, m=2$ to 4 .

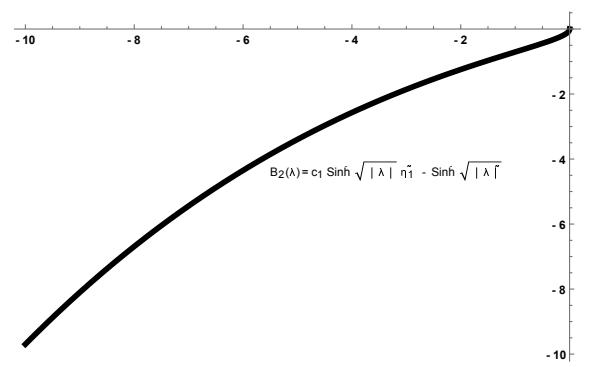

Figure 13. $B_{2}(\lambda)<0$.

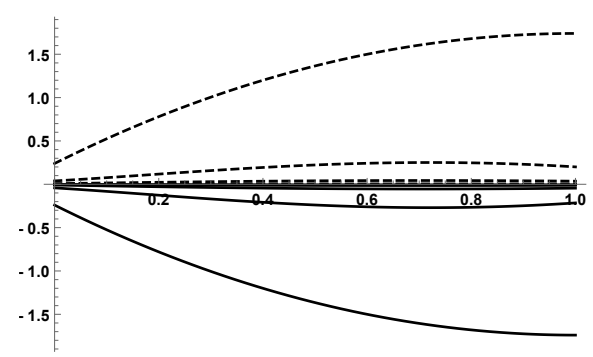

Figure 15. $\lambda=-1, m=5$.

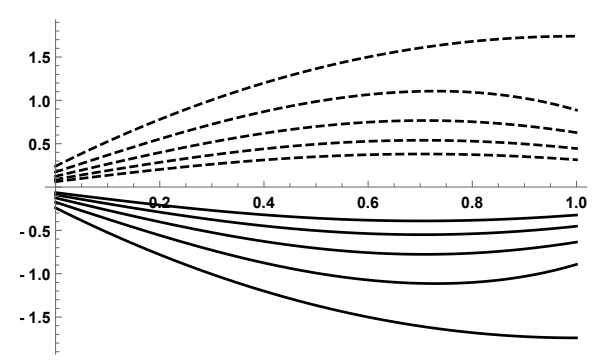

Figure 17. $\lambda=-10, m=5$.

\section{Conclusions}

The work of this paper generalises and complement our earlier work $[28,29]$. Since assumptions are simple we can deal with larger class of nonlinear four point boundary value problems (see [27, p. 27]) and since method is simple iterative, it is user friendly and can be used to develop software package to compute solutions of multi point BVPs.

\section{Acknowledgements}

We are thankful to all the reviewers for their valuable comments to improve the quality of the paper. We also thank to Bivek Gupta and Sheerin Kayenat for helping us in improving the English language of the paper. 


\section{References}

[1] M. Asaduzzaman and Md.Z. Ali. Fixed point theorem based solvability of fourth order nonlinear differential equation with four-point boundary value conditions. Adv. Fixed Point Theory, 9(3):260-272, 2019. https://doi.org/10.28919/afpt/4091.

[2] Md. Asaduzzaman. Existence results for a nonlinear fourth order ordinary differential equation with four-point boundary value conditions. Advances in the Theory of Nonlinear Analysis and its Application, 4(4):233-241, 2020. https://doi.org/10.31197/atnaa.774794.

[3] Md. Asaduzzaman, Md. Ali et al. On the symmetric positive solutions of nonlinear fourth order ordinary differential equations with fourpoint boundary value conditions: a fixed point theory approach. Journal of Nonlinear Sciences \& Applications (JNSA), 13(6):364-377, 2020. https://doi.org/10.22436/jnsa.013.06.06.

[4] Zh. Bai and Z. Du. Positive solutions for some second-order four-point boundary value problems. Journal of mathematical analysis and applications, 330(1):3450, 2007. https://doi.org/10.1016/j.jmaa.2006.07.044.

[5] Zh. Bai, W. Ge and Y. Wang. Multiplicity results for some second-order fourpoint boundary-value problems. Nonlinear Analysis: Theory, Methods \& Applications, 60(3):491-500, 2005. https://doi.org/10.1016/j.na.2004.08.039.

[6] Zh. Bai, W. Li and W. Ge. Existence and multiplicity of solutions for four-point boundary value problems at resonance. Nonlinear Analysis: Theory, Methods 86 Applications, 60(6):1151-1162, 2005. https://doi.org/10.1016/j.na.2004.10.013.

[7] P. Bailey, L.F. Shampine and P. Waltman. Nonlinear second order boundary value problems: Existence and regions of uniqueness. Journal of Mathematical Analysis and Applications, 14(3):433-444, 1966. https://doi.org/10.1016/0022247X(66)90004-7.

[8] P. Bailey, L.F. Shampine, P. Waltman et al. Existence and uniqueness of solutions of the second order boundary value problem. Bulletin of the American Mathematical Society, 72(1, Part 1):96-98, 1966. https://doi.org/10.1090/S0002-9904-1966-11434-9.

[9] I.V. Barteneva, A. Cabada and A.O. Ignatyev. Maximum and antimaximum principles for the general operator of second order with variable coefficients. Applied Mathematics and Computation, 134(1):173-184, 2003. https://doi.org/10.1016/S0096-3003(01)00280-6.

[10] A. Cabada. The method of lower and upper solutions for second, third, fourth, and higher order boundary value problems. Journal of Mathematical Analysis and Applications, 185(2):302-320, 1994. ISSN 0022-247X. https://doi.org/10.1006/jmaa.1994.1250.

[11] A. Cabada. An overview of the lower and upper solutions method with nonlinear boundary value conditions. Boundary value problems, 2011(1):893753, 2011. https://doi.org/10.1155/2011/893753.

[12] A. Cabada, P. Habets and S. Lois. Monotone method for the Neumann problem with lower and upper solutions in the reverse order. Applied Mathematics and Computation, 117(1):1-14, 2001. https://doi.org/10.1016/S00963003(99)00149-6. 
[13] M. Cherpion, C. De Coster and P. Habets. A constructive monotone iterative method for second-order BVP in the presence of lower and upper solutions. Applied Mathematics and Computation, 123(1):75-91, 2001. https://doi.org/10.1016/S0096-3003(00)00058-8.

[14] C. De Coster and P. Habets. Two-Point Boundary Value Problems: Lower and Upper Solutions, volume 205. Elsevier, 2006.

[15] P. Drábek, G. Holubová, A. Matas and P. Nečesal. Nonlinear models of suspension bridges: discussion of the results. Applications of Mathematics, 48(6):497514, 2003. https://doi.org/10.1023/B:APOM.0000024489.96314.7f.

[16] C.P. Gupta. A Dirichlet type multi-point boundary value problem for second order ordinary differential equations. Nonlinear Analysis: Theory, Methods 85 Applications, 26(5):925-931, 1996. https://doi.org/10.1016/0362-546X(94)00338$\mathrm{X}$.

[17] J. Henderson, S.K. Ntouyas and I.K. Purnaras. Positive solutions for systems of nonlinear discrete boundary value problems. Journal of Difference Equations and Applications, 15(10):895-912, 2009. https://doi.org/10.1080/10236190802350649.

[18] V.A. Ilyin and E.I. Moiseev. Nonlocal boundary value problem of the second kind for a Sturm Liouville operator on its differential and finite difference aspects. Differential Equations, 23(8):979-987, 1987.

[19] G. Infante. Nonlocal boundary value problems with two nonlinear boundary conditions. Communications in Applied Analysis, 12(3):279, 2008.

[20] G. Infante, F.M. Minhos and P. Pietramala. Non-negative solutions of systems of ODEs with coupled boundary conditions. Communications in Nonlinear Science and Numerical Simulation, 17(12):4952-4960, 2012. https://doi.org/10.1016/j.cnsns.2012.05.025.

[21] A.C. Lazer and P.J. McKenna. Large-amplitude periodic oscillations in suspension bridges: Some new connections with nonlinear analysis. SIAM Review, 32(4):537-578, 1990. https://doi.org/10.1137/1032120.

[22] B. Liu. Positive solutions of a nonlinear four-point boundary value problems. Applied Mathematics and Computation, 155(1):179-203, 2004. https://doi.org/10.1016/S0096-3003(03)00770-7.

[23] B. Liu. Positive solutions of a nonlinear four-point boundary value problems in Banach spaces. Journal of Mathematical Analysis and Applications, 305(1):253276, 2005. https://doi.org/10.1016/j.jmaa.2004.11.037.

[24] R. Ma and L. Ren. Positive solutions for nonlinear m-point boundary value problems of Dirichlet type via fixed-point index theory. Applied mathematics letters, 16(6):863-869, 2003. https://doi.org/10.1016/S0893-9659(03)90009-7.

[25] E. Picard. Mémoire sur la théorie des équations aux dérivées partielles et la méthode des approximations successives. Journal de Mathématiques pures et appliquées, 6:145-210, 1890.

[26] N. Urus, A.K. Verma and M. Singh. Some new existence results for a class of four point nonlinear boundary value problems. JNPG-The Journal of Revelations, 3:7-13, 2019.

[27] A.K. Verma, B. Pandit, L. Verma and R.P. Agarwal. A review on a class of second order nonlinear singular BVPs. Mathematics, 8(7):1045, 2020. https://doi.org/10.3390/math8071045. 
[28] A.K. Verma and M. Singh. A note on existence results for a class of three-point nonlinear BVPs. Mathematical Modelling and Analysis, 20(4):457-470, 2015. https://doi.org/10.3846/13926292.2015.1065293.

[29] A.K. Verma, N. Urus and M. Singh. Monotone iterative technique for a class of four point BVPs with reversed ordered upper and lower solutions. International Journal of Computational Methods, 17(9), 2020. https://doi.org/10.1142/S021987621950066X.

[30] L. Yang, C. Shen and Y. Liang. Existence, multiplicity of positive solutions for four-point boundary value problem with dependence on the first order derivative. Fixed Point Theory, 11(1):147-159, 2010.

[31] Y. Zhang. Positive solutions of singular sublinear Dirichlet boundary value problems. SIAM journal on mathematical analysis, 26(2):329-339, 1995. https://doi.org/10.1137/S0036141093246087.

\section{Appendix}

Here we provide the algorithm which we are using to compute the sequence of upper and lower solutions:

Step 1 Input the nonlinear term $F\left(x, \psi, \psi^{\prime}\right)$.

Step 2 Choose $m$ and $\lambda$, where $m=n$ is the number of members of sequences $\left(l_{m}\right)$ and $\left(u_{m}\right)$.

Step 3 Choose initial guesses : $l_{0}$ satisfying (4.1) and $u_{0}$ satisfying (4.1) with reverse inequalities.

Step 4 Start the iteration put $m=0$ in (4.2) and (4.3).

Step 5 Use $l_{0}, u_{0}$ and solve the resulting linear system to get $l_{1}$ and $u_{1}$.

Step 6 Repeat step 4 and step $5, n$ times to get $l_{0}, l_{1}, l_{2}, \ldots, l_{n}$ and $u_{0}, u_{1}, u_{2}$, $\ldots, u_{n}$.

Remark 1. We have used Mathematica 11.3 to solve the linearized multi point BVPs and plotting. Mathematica has DSolve and NDSolve command which can be used to solve the linear BVPs. 